\title{
CONDIÇÕES LMIS ALTERNATIVAS PARA SISTEMAS TAKAGI-SUGENO VIA FUNÇÃO DE LYAPUNOV FUZZY
}

\author{
Leonardo Amaral Mozelli* \\ mozelli@cpdee.ufmg.br \\ Gustavo Silva Castilho de Avellar* \\ gustavoavellar@yahoo.com.br
}

\author{
Reinaldo Martinez Palhares $\dagger^{*}$ \\ palhares@cpdee.ufmg.br \\ Rafael Ferreira dos Santos* \\ rafaelfds@yahoo.com.br
}

\author{
*Universidade Federal de Minas Gerais \\ Departamento de Engenharia Eletrônica \\ Av. Antônio Carlos, 6627 - Pampulha \\ 31270-010 - Belo Horizonte - Minas Gerais - Brasil \\ $\dagger$ Autor para contato.
}

\begin{abstract}
Alternative LMI Conditions for Takagi-Sugeno Systems Via Fuzzy Lyapunov Function

This paper deals with the stability analysis and control design for continuous Takagi-Sugeno fuzzy systems in a linear matrix inequality (LMI) framework. New LMI stability conditions are obtained by applying a relaxation strategy in a recently proposed fuzzy Lyapunov function. In these new LMI stability conditions, new degrees of freedom are introduced, such that the conservativeness can be reduced. Furthermore, these conditions allow to design the control law entirely described by LMIs, which is much more attractive than bilinear matrix inequalities (BMI) approaches available in the literature. Numerical tests and simulations illustrate the advantages of the new methodology.
\end{abstract}

KEYWORDS: fuzzy Lyapunov function, Takagi-Sugeno (TS) fuzzy model, linear matrix inequalities (LMIs), fuzzy control

\footnotetext{
Artigo submetido em 30/03/2009 (Id.: 00982)

Revisado em 29/05/2009, 12/08/2009

Aceito sob recomendação do Editor Associado Prof. Alexandre Bazanella
}

\section{RESUMO}

Este trabalho versa sobre análise de estabilidade e projeto de controladores para sistemas fuzzy Takagi-Sugeno (TS) contínuos no contexto de desigualdades matriciais lineares (LMIs). Aplicando uma estratégia de relaxação a um tipo de função de Lyapunov fuzzy proposta recentemente, é possível obter novas condições de estabilidade descritas por LMIs. Essas novas condições conferem novos graus de liberdade ao problema LMI, reduzindo o conservadorismo. Mais do que isso, tal estratégia permite a síntese de controladores inteiramente por meio de LMIs, uma característica bem mais atrativa do que os procedimentos baseados em desigualdades matriciais bilineares (BMIs) presentes na literatura. Teste numéricos e simulações ilustram as vantagens da nova metodologia.

PALAVRAS-CHAVE: função de Lyapunov fuzzy, modelagem fuzzy Takagi-Sugeno (TS), desigualdades matriciais lineares (LMIs), controle fuzzy

\section{INTRODUÇÃO}

Tradicionalmente, a análise de estabilidade e a síntese de controladores para sistemas fuzzy Takagi-Sugeno (TS) (Takagi e Sugeno, 1985) são baseadas na busca de uma 
função quadrática capaz de garantir um certificado de estabilidade global no sentido de Lyapunov para o sistema, como proposto inicialmente por Tanaka e Sugeno (1992).

O método direto de Lyapunov estabelece condições suficientes para estabilidade, que podem ser reescritas como desigualdades matriciais lineares (LMIs, acrônimo inglês para linear matrix inequalities), problemas de otimização convexa cujas soluções podem ser obtidas por meio de diversos pacotes numéricos (Gahinet et al., 1995; Löfberg, 2004).

No entanto, determinar uma única função de Lyapunov quadrática (FLQ) pode não ser viável, especialmente no caso de um grande número de regras (Tanaka et al., 1998). Com essa motivação, diversos trabalhos buscaram reduzir o conservadorismo das condições LMI baseadas na FLQ por meio da introdução de variáveis de folga (Tanaka et al., 1998; Kim e Lee, 2000; Teixeira et al., 2000; Teixeira et al., 2003; Liu e Zhang, 2003; Arrifano e Oliveira, 2004; Fang et al., 2006; Mozelli et al., 2007; Andrea et al., 2008).

Recentemente, nos trabalhos independentes de Sala e Ariño (2007) e Montagner et al. (2007), (2009) foram propostas condições teóricas para determinar de forma necessária e suficiente a existência de uma FLQ, demarcando a fronteira para o desempenho quadrático. Numericamente, as condições propostas em (Montagner et al., 2009) se traduzem como sequencias convergentes de LMIs, cujo conservadorismo diminui à medida que certo parâmetro cresce, tendendo para a condição de necessidade.

Contudo, um sistema TS pode ser estável mesmo inexistindo uma FLQ para certificá-la, como demonstrado por Johansson et al. (1999). Nesse caso, existem funções de Lyapunov alternativas que dão garantia de estabilidade global, dentre as quais destacam-se a chamada função de Lyapunov fuzzy (FLF) (Tanaka et al., 2003; Tanaka et al., 2007; Mozelli et al., 2008; Mozelli et al., 2009), composta pela interpolação de funções quadráticas segundo as mesmas funções de pertinência usadas na modelagem TS, e a função de Lyapunov por partes (Johansson et al., 1999; Arrifano et al., 2006).

Resultados menos conservadores são obtidos para projeto de controladores usando a FLF proposta em (Tanaka et al., 2003). Contudo, destaca-se a presença da derivada temporal das funções de pertinência na variação temporal da função. Para obter condições LMI é preciso selecionar um limitante superior para a derivada das funções de pertinência. Embora métodos analíticos para obter esse limitante estejam disponíveis (Tanaka et al., 2003; Tanaka et al., 2007) e o uso dessa informação possa reduzir conservadorismo, como mostrado em (Mozelli et al., 2009), nem sempre seu cálculo pode ser realizado.

Outro fator que pesa contra a FLF na forma proposta por Tanaka et al. (2003) é o fato de que a síntese de controladores é feita segundo desigualdades matriciais bilineares (BMIs, acrônimo do inglês, bilinear matrix inequalities ), cuja solução é mais lenta e pode acarretar em conservadorismo extra. Em (Tanaka et al., 2007; Mozelli et al., 2008) são propostas alternativas para síntese de controladores através de LMIs, mas a questão sobre o limitante da derivada permanece.

Rhee e Won (2006) propõem uma FLF alternativa cuja diferenciação no tempo não depende das funções de pertinência e pode levar a condições de estabilidade e estabilização menos conservadoras que em (Tanaka et al., 2003). Contudo, eliminar a informação da pertinência conduz a matrizes da função de Lyapunov que possuem características especiais (além de simetria), fazendo com que o projeto do controlador recaia outra vez em condições BMIs. Isso é menos vantajoso, haja vista o procedimento proposto por Rhee e Won (2006) que é baseado em um algoritmo iterativo bastante elaborado, que inclui a escolha ad hoc de alguns parâmetros para garantir convergência, o que torna a sua reprodução difícil.

Na versão inicial deste trabalho (de Avellar et al., 2008) condições LMI alternativas foram obtidas por meio de uma abordagem sistemática capaz de introduzir variáveis de folga, estratégia bem sucedida no contexto de análise de estabilidade para certas classes de sistemas não-lineares com retardo no tempo (Souza, Palhares e Ekel, 2008; Souza, Palhares, Mendes e Tôrres, 2008a; Souza, Palhares, Mendes e Tôrres, 2008b; Souza et al., 2009). Tais variáveis, reconhecidas também como multiplicadores matriciais (de Oliveira, 2004), permitem o desacoplamento das matrizes da FLF com relação às matrizes do sistema, conferindo novos graus de liberdade ao problema de otimização. Isso permitiu obter uma abordagem para síntese de controlador baseada em LMIs, ao contrário da abordagem via BMIs de (Rhee e Won, 2006).

Este artigo apresenta como novidades em relação a (de Avellar et al., 2008): i) aperfeiçoar as condições LMI para estabilidade por meio da introdução de mais variáveis de folga (multiplicadores e termos de relaxação do lado direito); ii) aprimorar a abordagem LMI para síntese de controladores, eliminando restrições que existiam sobre os multiplicadores matriciais. 
Notação: O sobre-escrito $T$ indica transposto para matrizes e vetores; o sobre-escrito $-T$ indica a inversa da matriz transposta; a notação $(*)$ é usada para substituir termos transpostos em uma matriz simétrica; $\operatorname{sim}(X)$ indica $X+X^{T}$; já $\operatorname{diag}(\cdot)$ indica uma matriz diagonal; os subconjuntos $\{1,2, \ldots, r\} \subset \mathbb{N}$ e $\{1,2, \ldots, n\} \subset \mathbb{N}$ são denotados por $\mathcal{R}$ e $\mathcal{N}$, respectivamente. Para maior simplificação adota-se:

$$
\sum_{i=1}^{r} \sum_{\substack{j=1 \\ i<j}}^{r} h_{i j} \Leftrightarrow \sum_{i<j}^{r} h_{i j}
$$

Por simplicidade, adota-se a seguinte notação nas demonstrações dos teoremas: $x(t) \Leftrightarrow x \quad \beta_{i}(x(t)) \Leftrightarrow \beta_{i}$.

\section{MODELO FUZZY TAKAGI-SUGENO}

Considere o seguinte modelo fuzzy TS para sistemas dinâmicos não-lineares contínuos (Rhee e Won, 2006):

$$
\begin{gathered}
R_{i}: \quad \operatorname{Se~} x_{1}(t) \text { é } \mathcal{M}_{1}^{\alpha_{i 1}} \text { e } \cdots \text { e } x_{n}(t) \text { é } \mathcal{M}_{n}^{\alpha_{i n}} \\
\text { ENT̃̃o } \dot{x}(t)=A_{i} x(t)+B_{i} u(t)
\end{gathered}
$$

sendo o vetor de estados $x(t) \in \mathbb{R}^{n}$; a entrada de controle é indicada por $u(t) \in \mathbb{R}^{m}$; o número de modelos locais e regras $R_{i}$ é dado por $r ; A_{i}, B_{i}$ são matrizes reais de dimensão apropriada; para esse modelo, os estados $x(t)$ são escolhidos como variáveis premissas; os conjuntos fuzzy são indicados por $\mathcal{M}_{j}^{\alpha_{i j}}, i \in \mathcal{R}, j \in \mathcal{N}$. Para maiores detalhes veja (Rhee e Won, 2006). O modelo inferido a partir de (1) é indicado de forma compacta por:

$$
\dot{x}(t)=A(x) x(t)+B(x) u(t),
$$

sendo

$$
A(x) \triangleq \sum_{i=1}^{r} \beta_{i}(x(t)) A_{i}, \quad B(x) \triangleq \sum_{i=1}^{r} \beta_{i}(x(t)) B_{i},
$$

e $\beta_{i}(x(t))$ são as funções de pertinência normalizadas que satisfazem:

$$
0 \leq \beta_{i}(x(t)) \leq 1, \sum_{i=1}^{r} \beta_{i}(x(t))=1, i \in \mathcal{R} .
$$

Nota 1 Para o leitor com interesse nas etapas de modelagem de sistemas fuzzy TS, recomenda-se que consulte as referências: Tanaka e Wang (2001), Andrea et al. (2008), Mozelli et al. (2007), Teixeira e Assunção (2007), Tanscheit et al. (2007), Khiar et al. (2007), Arrifano e Oliveira (2004), Teixeira et al. (2000) com inúmeros exemplos detalhados.

\section{FUNÇÃO DE LYAPUNOV FUZZY}

A função de Lyapunov fuzzy que será utilizada neste artigo para estabelecer novas condições de estabilidade e estabilização foi proposta por (Rhee e Won, 2006), dada segundo:

$$
V(x(t))=2 \int_{\Gamma(0, x)} P(\psi) \psi d \psi,
$$

sendo que $\Gamma(0, x)$ é um caminho da origem até o estado atual, $\psi$ é um vetor para a integração e $d \psi$ é um vetor de deslocamento infinitesimal. A função $P(x)$ é parametrizada de acordo com as mesmas pertinências do modelo TS (3):

$$
P(x)=\sum_{i=1}^{r} \beta_{i}(x(t)) P_{i}
$$

Portanto a função (4) também recai na categoria de funções de Lyapunov fuzzy, pois depende da interpolação fuzzy de matrizes constantes $P_{i}$ por meio das pertinên$\operatorname{cias} \beta_{i}(x(t))$.

Funções de Lyapunov no formato de integral são utilizadas no contexto geral de sistemas não-lineares (Haddad e Chellaboina, 2008). Em geral, tais funções não são construídas através do método de Krasovskii (Haddad e Chellaboina, 2008, Cap. 3), no qual se determina uma função candidata quadrática definida positiva (ou uma combinação fuzzy delas como em (Tanaka et al., 2003)) cuja derivada ao longo das trajetórias do sistema é uma função definida negativa. Alternativamente, a construção daquelas funções se dá pelo método do gradiente variável (Haddad e Chellaboina, 2008, Cap. 3), no qual assume-se que $g(x)$ é o gradiente de uma função de Lyapunov desconhecida que satisfaz $g(x)^{T} \dot{x}<0$. Caso a matriz Jacobiana $\partial g(x) / \partial x$ seja simétrica, então a função de Lyapunov desconhecida é construída como a integral de linha de $g(x)$. Uma vez obtida a função de Lyapunov resta verificar se é definida positiva.

Assumindo que o gradiente da função desconhecida é dado como uma combinação fuzzy de matrizes constantes, sem depender da derivada temporal dessas pertinências, tem-se que

$$
g(x)^{T} \dot{x}=2 x(t)^{T} P(x) \dot{x}(t) .
$$

Para garantir a condição de simetria do Jacobiano de $\partial g(x) / \partial x$ as matrizes $P_{i}$ têm a forma especial a seguir, veja detalhes (Rhee e Won, 2006): 


$$
P_{i} \triangleq D_{0}+D_{i},
$$

sendo

$$
\begin{gathered}
D_{0} \triangleq\left[\begin{array}{cccc}
0 & d_{12} & \cdots & d_{1 n} \\
d_{12} & 0 & \cdots & d_{2 n} \\
\vdots & \vdots & \ddots & \vdots \\
d_{1 n} & d_{2 n} & \cdots & 0
\end{array}\right], \\
D_{i} \triangleq\left[\begin{array}{cccc}
d_{11}^{\alpha_{i 1}} & 0 & \cdots & 0 \\
0 & d_{22}^{\alpha_{i 2}} & \cdots & 0 \\
\vdots & \vdots & \ddots & \vdots \\
0 & 0 & \cdots & d_{n n}^{\alpha_{i n}}
\end{array}\right] .
\end{gathered}
$$

Esta notação indica que somente os elementos de $D_{i}$ (elementos da diagonal de $P_{i}$ ) mudam com as regras fuzzy. Os elementos da diagonal são alterados de acordo com os conjuntos fuzzy na premissa da regras $\mathrm{SE}-$ EnTÃo. Se uma variável premissa pertencer ao mesmo conjunto fuzzy em regras distintas, então os elementos da diagonal relativos àquela variável serão iguais. Contudo, seja $x_{j}(t)$ pertencente a conjuntos distintos nas regras $k$ e $l$. Os $j$-ésimos elementos da diagonal de $D_{k}$ e $D_{l}$ (quais sejam $d_{j j}^{\alpha_{k j}}$ e $d_{j j}^{\alpha_{l j}}$ ) serão diferentes.

Rhee e Won (2006) demonstraram em detalhes que (4) é uma função de Lyapunov se as matrizes $P_{i}$ possuem a estrutura apresentada em (6). Nota-se contudo que a demonstração não segue as idéias apresentadas nessa seção, baseadas no método do gradiente variável.

\section{NOVAS CONDIÇÕES DE ESTABILI- DADE}

Nesta seção, as novas condições de estabilidade para sistemas TS contínuos serão enunciadas. Essas condições visam reduzir ainda mais o grau de conservadorismo apresentado em (Rhee e Won, 2006) com o uso de mais matrizes de folga. Antes disso, a condição original apresentada em (de Avellar et al., 2008) é apresentada.

Teorema 1 O sistema fuzzy contínuo (2) é estável se existirem matrizes $P_{i}$, com a estrutura dada em (6), e matrizes $M$ e $G$ quaisquer, que satisfaçam as seguintes LMIs:

$$
\begin{aligned}
& P_{i} \succ 0, \\
& \Theta_{i} \prec 0 \quad i \in \mathcal{R}
\end{aligned}
$$

sendo

$$
\Theta_{i} \triangleq\left[\begin{array}{cc}
-M A_{i}-A_{i}^{T} M^{T} & * \\
P_{i}-G A_{i}+M^{T} & G+G^{T}
\end{array}\right] .
$$

Prova: Considere (7) e assuma (4) como a função de Lyapunov candidata. Aplicando a derivada de Lie:

$$
\dot{V}(x)=\sum_{i=1}^{r} \beta_{i}\left\{x^{T} P_{i} \dot{x}+\dot{x}^{T} P_{i} x\right\} .
$$

Caso $u(t)=0 \mathrm{em}(2)$, a identidade a seguir é verdadeira:

$$
2\left[x^{T} M+\dot{x}^{T} G\right] \times[\dot{x}-A(x) x]=0 .
$$

Adicionando (11) a (10) e em seguida utilizando o vetor a seguir:

$$
\xi \triangleq\left[\begin{array}{ll}
x^{T} & \dot{x}^{T}
\end{array}\right]^{T},
$$

tem-se que:

$$
\dot{V}(x)=\sum_{i=1}^{r} \beta_{i} \xi^{T} \Theta_{i} \xi,
$$

sendo $\Theta_{i}$ definido em (9). Se (8) é satisfeita, então $\dot{V}(x)<0$. Portanto, conclui-se da teoria de Lyapunov que a estabilidade global do sistema TS (2) é garantida.

No Teorema 1, foram introduzidas as matrizes de folga $M$ e $G$. Uma nova versão desse teorema envolvendo mais matrizes também é proposta a seguir.

Teorema 2 O sistema fuzzy contínuo (2) é estável se existirem matrizes $P_{i}$, com a estrutura dada em (6), e matrizes $M_{i}$ e $G_{i}$ quaisquer, que satisfaçam as seguintes LMIs:

$$
\begin{aligned}
& P_{i} \succ 0, \\
& \Theta_{i i} \prec 0, \\
& \Theta_{i j}+\Theta_{j i} \prec 0, i, j \in \mathcal{R}, i<j
\end{aligned}
$$

sendo

$$
\Theta_{i j} \triangleq\left[\begin{array}{cc}
-M_{j} A_{i}-A_{i}^{T} M_{j}^{T} & * \\
P_{i}-G_{j} A_{i}+M_{j}^{T} & G_{j}+G_{j}^{T}
\end{array}\right] .
$$

Prova: Segue passos similares aos da demonstração do Teorema 1. Contudo a identidade a seguir é considerada no lugar de (11):

$$
2\left[x^{T} M(x)+\dot{x}^{T} G(x)\right] \times[\dot{x}-A(x) x]=0,
$$

sendo

$$
M(x)=\sum_{i=1}^{r} \beta_{i} M_{i}, \quad G(x)=\sum_{i=1}^{r} \beta_{i} G_{i},
$$


para se obter

$$
\begin{aligned}
\dot{V}(x) & =\sum_{i=1}^{r} \sum_{j=1}^{r} \beta_{i} \beta_{j} \xi^{T} \Theta_{i j} \xi \\
& =\sum_{i=1}^{r} \beta_{i}^{2} \xi^{T} \Theta_{i i} \xi+\sum_{i<j}^{r} \beta_{i} \beta_{j} \xi^{T}\left(\Theta_{i j}+\Theta_{j i}\right) \xi
\end{aligned}
$$

sendo $\Theta_{i j}$ definido em (17).

Caso (15) e (16) sejam satisfeitas, então o sistema é globalmente assintoticamente estável.

Note que nos Teoremas 1 e 2 as matrizes $A_{i}$ encontramse separadas das matrizes $P_{i}$, ao contrário do que ocorre nas condições propostas por Rhee e Won (2006). É interessante constatar também que para agregar uma maior quantidade de multiplicadores matriciais ao Teorema 2 foi preciso adicionar mais restrições ao problema. Essas restrições extras, na forma de somatório duplo como em (19), permitem que mais matrizes de folga sejam adicionadas usando, por exemplo, a estratégia apresentada por (Liu e Zhang, 2003). Combinando essa estratégia é possível obter as novas condições a seguir:

Teorema 3 O sistema fuzzy contínuo (2) é estável se existirem matrizes $P_{i}$, com a estrutura dada em (6), matrizes simétricas $Y_{i i}$ e matrizes $M_{i}, G_{i}$ e $Y_{j i}=Y_{i j}^{T}$ quaisquer, que satisfaçam as seguintes LMIs:

$$
\begin{aligned}
& P_{i} \succ 0, \\
& \Theta_{i i} \prec Y_{i i}, \\
& \Theta_{i j}+\Theta_{j i} \preceq Y_{i j}+Y_{j i}, i, j \in \mathcal{R}, i<j \\
& \bar{Y}=\left[\begin{array}{cccc}
Y_{11} & Y_{21}^{T} & \cdots & Y_{r 1}^{T} \\
Y_{21} & Y_{22} & \cdots & Y_{r 2}^{T} \\
\vdots & \vdots & \ddots & \vdots \\
Y_{r 1} & Y_{r 2} & \cdots & Y_{r r}
\end{array}\right] \prec 0
\end{aligned}
$$

sendo que $\Theta_{i j}$ é definido como em (17).

Prova: A demonstração do Teorema 2 é tomada como ponto de partida. Considerando (19), caso (21) e (22) sejam satisfeitas, tem-se que

$$
\begin{gathered}
\dot{V}(x) \leq \sum_{i=1}^{r} \beta_{i}^{2} \xi^{T} Y_{i i} \xi+\sum_{i<j}^{r} \beta_{i} \beta_{j} \xi^{T}\left(Y_{i j}+Y_{j i}\right) \xi \\
=\left[\begin{array}{c}
\beta_{1} \xi \\
\beta_{2} \xi \\
\vdots \\
\beta_{r} \xi
\end{array}\right]^{T}\left[\begin{array}{cccc}
Y_{11} & Y_{21}^{T} & \cdots & Y_{r 1}^{T} \\
Y_{21} & Y_{22} & \cdots & Y_{r 2}^{T} \\
\vdots & \vdots & \ddots & \vdots \\
Y_{r 1} & Y_{r 2} & \cdots & Y_{r r}
\end{array}\right]\left[\begin{array}{c}
\beta_{1} \xi \\
\beta_{2} \xi \\
\vdots \\
\beta_{r} \xi
\end{array}\right]
\end{gathered}
$$

Se $(23)$ é satisfeita, então $\dot{V}(x)<0$, garantindo a estabilidade global do sistema TS (2).

A comparação entre os Teoremas propostos com respeito à quantidade de variáveis de folga e de LMIs pode ser vista na Tabela 1. Pode-se afirmar que há um compromisso entre custo computacional e desempenho. Isso ocorre pois em geral uma maior quantidade de variáveis de folga tende a tornar as condições menos conservadoras. Todavia uma maior quantidade de LMIs e de variáveis de folga acarreta em maior tempo de execução do problema.

Tabela 1: Número de LMIs $(l)$ e de variáveis de folga $(v)$ em alguns métodos baseados em FLF.

\begin{tabular}{ccc}
\hline Método & $l$ & $v$ \\
\hline (Rhee e Won, 2006) & $\frac{r^{2}+3 r+2}{2}$ & 1 \\
Teorema 1 & $2 r$ & 2 \\
Teorema 2 & $\frac{r^{2}+3 r}{2}$ & $2 r$ \\
Teorema 3 & $\frac{r^{2}+3 r+2}{2}$ & $\frac{r^{2}+5 r}{2}$
\end{tabular}

\section{EXEMPLO DE ANÁLISE DE ESTABILI- DADE}

O exemplo a seguir é utilizado para demonstrar como as condições propostas reduzem o conservadorismo presente em abordagens baseadas em FLQ (Tanaka e Sugeno, 1992; Tanaka et al., 1998; Kim e Lee, 2000; Liu e Zhang, 2003; Teixeira et al., 2003; Fang et al., 2006; Sala e Ariño, 2007; Montagner et al., 2007; Montagner et al., 2009) e também na abordagem original em (Rhee e Won, 2006). Neste exemplo, o sistema analisado possui três regras

$$
\begin{array}{lll}
R_{1}: & \text { SE } x_{1}(t) \text { é } \mathcal{M}_{1}^{1} & \text { ENTÃo } \dot{x}(t)=A_{1} x(t) \\
R_{2}: & \text { SE } x_{1}(t) \text { é } \mathcal{M}_{1}^{2} & \text { ENTÃo } \dot{x}(t)=A_{2} x(t) \\
R_{3}: & \text { SE } x_{1}(t) \text { é } \mathcal{M}_{1}^{3} & \text { ENTÃo } \dot{x}(t)=A_{3} x(t)
\end{array}
$$

As matrizes locais são dadas por:

$$
A_{1}=\left[\begin{array}{cc}
0.00 & a \\
-0.06 & -1.00
\end{array}\right], A_{2}=\left[\begin{array}{cc}
0.00 & 1.00 \\
-1.94 & -1.00
\end{array}\right] \text {, }
$$




$$
A_{3}=\left[\begin{array}{cc}
0.00 & b \\
-0.50 & -1.50
\end{array}\right]
$$

As matrizes de Lyapunov para este exemplo devem ser montadas da seguinte forma (veja a definição em 6):

$$
\begin{gathered}
P_{1}=\left[\begin{array}{ll}
d_{11}^{1} & p_{21} \\
p_{21} & p_{22}
\end{array}\right], P_{2}=\left[\begin{array}{ll}
d_{11}^{2} & p_{21} \\
p_{21} & p_{22}
\end{array}\right], \\
P_{3}=\left[\begin{array}{ll}
d_{11}^{3} & p_{21} \\
p_{21} & p_{22}
\end{array}\right],
\end{gathered}
$$

pois há apenas uma variável premissa $x_{1}(t)$ que pertence a um conjunto diferente em cada uma das regras. Os intervalos nos quais a estabilidade foi analisada são $a \in$ $[-0.25,5]$ e $b \in[-0.25,7]$.

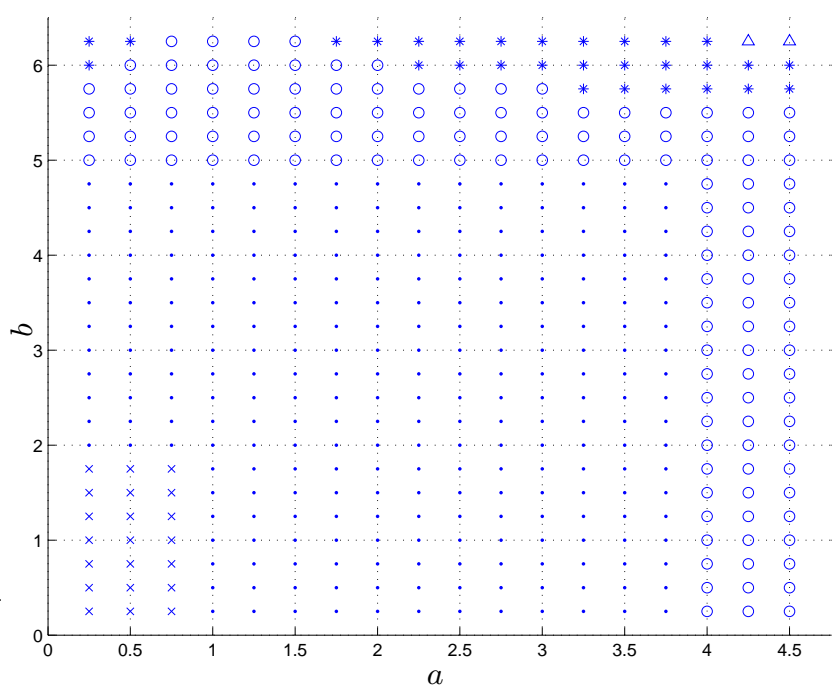

Figura 1: Regiões de estabilidade geradas pelas abordagens baseadas em FLQ e FLF. (Fang et al., 2006) $\rightarrow(\times)$; (Rhee e Won, 2006) $\rightarrow(\times)$ e $(\cdot)$; Teorema $1 \rightarrow(\times)$, (.) e (o); Teorema $2 \rightarrow(\times),(\cdot),(\circ)$ e $(*)$; Teorema 3 inclui todos os pontos assinalados na Figura.

A Figura 1 exibe o resultado obtido através da abordagem baseada em FLQ em (Fang et al., 2006), onde os pares $a$ e $b$ assinalados com $(\times)$ representam sistemas estáveis. O resultado obtido por (Rhee e Won, 2006) está marcado por $(\times)$ e $(\cdot)$.

O Teorema 1 corresponde aos pontos marcados por $(\times)$, (.) e (o). O Teorema 2 inclui o resultado do Teorema 1 e ainda os pontos assinalados por $(*)$. Finalmente, o resultado do Teorema 3 inclui todos os pontos assinalados na Figura 1.

É fácil constatar, através da Figura 1, que mesmo abordagens FLQ que usam um grande número de variáveis de folga, como (Fang et al., 2006) podem ser mais conservadoras que as abordagens baseadas em FLF.
Por outro lado, analisando a Figura 1, nota-se facilmente que as abordagens propostas neste artigo são bem menos conservadoras que a abordagem proposta por Rhee e Won (2006). Mesmo o Teorema 1, que apresenta apenas 1 variável de folga a mais que as condições de (Rhee e Won, 2006), é capaz de expandir a região factível, com a vantagem de utilizar uma quantidade de LMIs que cresce de maneira linear com o número de regras (veja a Tabela 1), ao passo que (Rhee e Won, 2006) demanda uma quantidade que aumenta quadraticamente.

O resultado desse exemplo permite ainda conjecturar que condições menos conservadoras podem ser obtidas seguindo a metodologia apresentada em (Montagner et al., 2007; Montagner et al., 2009). Note que o Teorema 5 usa uma combinação de matrizes dada segundo

$Y(x)=\sum_{i=1}^{r} \beta_{i}(x(t)) Y_{i i}+\sum_{i=1}^{r} \sum_{j=1}^{r} \beta_{i}(x(t)) \beta_{j}(x(t))\left(Y_{i j}+Y_{j i}\right)$,

para majorar as condições do Teorema 4. Nesse caso, seguindo as idéias propostas em (Sala e Ariño, 2007; Montagner et al., 2007; Montagner et al., 2009) seria possível substituir $Y(x)$ por um polinômio homogêneo genérico como relaxação do lado direito. Analogamente, as matrizes de desacoplamento $G(x)$ e $M(x)$ podem ser trocadas por polinômios homogêneos de grau maior do que 1.

\section{PROJETO DE CONTROLADOR}

Nesta seção serão apresentados novos procedimentos baseados em LMIs para fazer a síntese de controladores fuzzy. É utilizado o conceito de compensação paralela distribuída (PDC) (Tanaka et al., 1998; Teixeira et al., 2000; Rhee e Won, 2006), em que o controlador baseia-se no mesmo conjunto de regras (1) do modelo TS (2):

$$
u(t)=F(x) x(t),
$$

sendo que

$$
F(x) \triangleq \sum_{i=1}^{r} \beta_{i}(x(t)) F_{i},
$$

e $F_{i} \in \mathbb{R}^{m \times n}$ são os ganhos locais.

Considerando a realimentação de estados (25), o modelo TS (2) em malha fechada é descrito da forma compacta:

$$
\dot{x}(t)=[A(x)+B(x) F(x)] x(t) .
$$

Neste ponto vale destacar um aspecto relativo às condições propostas por (Rhee e Won, 2006) que tornam a 
síntese de controladores não trivial. Da teoria de Lyapunov, para garantir estabilidade global usando a função (4), é suficiente satisfazer:

$$
\begin{aligned}
& P(x) \succ 0, \\
& \operatorname{sim}(P(x)[A(x)+B(x) F(x)] x(t)) \prec 0 .
\end{aligned}
$$

Uma estratégia comum no caso de estabilidade quadrática (Tanaka et al., 1998; Teixeira et al., 2000) para linearizar esse problema, que possui o produto das variáveis $P(x)$ e $F(x)$, consiste em aplicar uma transformação de similaridade. No caso específico das desigualdades acima, essa transformação consistiria em multiplicá-las a direita e a esquerda por $P(x)^{-1}$. Tal inversa existe, dado que $P(x) \succ 0$, então obter-se-iam as novas desigualdades:

$$
\begin{aligned}
& P^{-1}(x) \succ 0, \\
& \operatorname{sim}\left([A(x)+B(x) F(x)] P^{-1}(x) x(t)\right) \prec 0 .
\end{aligned}
$$

Esse último conjunto de desigualdades é um problema de dimensão infinita com relação às variáveis premissas (Lian et al., 2006). Todavia, dadas as propriedades das funções de pertinência, veja (3), esse é um problema convexo, portanto seria equivalente a um número finito de restrições do tipo:

$$
\begin{aligned}
& P_{k}^{-1} \succ 0, \\
& \operatorname{sim}\left(\left[A_{i}+B_{i} F_{j}\right] P_{k}^{-1}\right) \prec 0 .
\end{aligned}
$$

Desta forma, bastaria definir $\Phi_{j k}=F_{j} P_{k}^{-1}$ como novas variáveis, linearizando o problema.

Contudo, dois aspectos inviabilizam a aplicação desse expediente. De posse de $\Phi_{j k}$ que satisfazem a desigualdade não seria possível determinar unicamente $F_{j}$, haja vista que não se pode garantir que $\Phi_{1 j} P_{1}=\Phi_{2 j} P_{2}$.

Outro aspecto com relação a essa transformação diz respeito à estrutura imposta sobre a matriz $P(x)$, relembre a definição 6. Por exemplo, considere o caso no qual tem-se matrizes $P_{1}$ e $P_{2}$ com os termos fora da diagonal idênticos. Ao se inverter essas matrizes não se pode garantir que $P_{1}^{-1}$ e $P_{2}^{-1}$ ainda têm os termos fora da diagonal iguais. Portanto, conclui-se que a extensão dos resultados de (Rhee e Won, 2006) para formulações puramente LMIs não é direta.

O próximo Teorema apresenta um procedimento para síntese de controladores que é inteiramente baseado em
LMIs, ao contrário do procedimento apresentado por Rhee e Won (2006), que é baseado em BMIs. Esse Teorema apresenta condições suficientes para obtenção dos ganhos $F_{i}$ para estabilização do sistema em malha fechada (26).

Teorema 4 Considere um escalar $\mu>0$ dado. O sistema (26) é estabilizável pelo controlador (25), com ganhos locais dados por $F_{i}=S_{i} R^{-1}$, se existirem matrizes $P_{i}$, com a estrutura dada em (6), e matrizes $R, S_{i}$ quaisquer tais que as LMIs abaixo sejam factíveis:

$$
\begin{aligned}
& P_{i} \succ 0, \\
& \tilde{\Theta}_{i i} \prec 0, \\
& \bar{\Theta}_{i j} \prec 0, \quad i, j \in \mathcal{R}, i<j
\end{aligned}
$$

sendo

$$
\begin{aligned}
\tilde{\Theta}_{i j} \triangleq & {\left[\begin{array}{cc}
\tilde{\theta}_{11}^{i j} & * \\
\tilde{\theta}_{21}^{i j} & \tilde{\theta}_{22}^{i j}
\end{array}\right], \quad \bar{\Theta}_{i j} \triangleq\left[\begin{array}{cc}
\bar{\theta}_{11}^{i j} & * \\
\bar{\theta}_{21}^{i j} & \bar{\theta}_{22}^{i j}
\end{array}\right] } \\
\tilde{\theta}_{11}^{i j} & \triangleq-A_{i} R-R^{T} A_{i}^{T}-B_{i} S_{j}-S_{j}^{T} B_{i}^{T} \\
\tilde{\theta}_{21}^{i j} & \triangleq P_{i}-\mu\left(A_{i} R+B_{i} S_{j}\right)+R^{T} \\
\tilde{\theta}_{22}^{i j} & \triangleq \mu\left(R+R^{T}\right), \quad \bar{\theta}_{a b}^{i j} \triangleq \tilde{\theta}_{a b}^{i j}+\tilde{\theta}_{a b}^{j i}
\end{aligned}
$$

Prova: Primeiramente, reescreve-se (10) como

$$
\dot{V}(x)=\sum_{i=1}^{r} \beta_{i} \xi^{T} \Lambda_{i} \xi
$$

sendo $\xi$ definido em (12) e

$$
\Lambda_{i} \triangleq\left[\begin{array}{cc}
0 & P_{i} \\
P_{i} & 0
\end{array}\right]
$$

A identidade a seguir

$$
2\left[x^{T} M^{-1}+\dot{x}^{T} G^{-1}\right] \times[\dot{x}-\bar{A}(x) x]=0
$$

na qual $\bar{A}(x) \triangleq A(x)+B(x) F(x)$, também pode ser colocada na forma

$$
\sum_{i=1}^{r} \sum_{j=1}^{r} \beta_{i} \beta_{j} \xi^{T} \Gamma_{i j} \xi=0
$$

sendo

$$
\Gamma_{i j} \triangleq\left[\begin{array}{cc}
\operatorname{sim}\left(-M^{-1} A_{i}-M^{-1} B_{i} F_{j}\right) & * \\
M^{-T}-G^{-1} A_{i}-G^{-1} B_{i} F_{j} & \operatorname{sim}\left(G^{-T}\right)
\end{array}\right] .
$$


Faça a escolha particular $G^{-1}=\mu M^{-1}$, tal que $\mu$ é um escalar positivo. Multiplicando $\Gamma_{i j}$ a esquerda e a direita por $\operatorname{diag}(M, M)$ e $\operatorname{diag}\left(M^{T}, M^{T}\right)$, respectivamente, tem-se

$$
\bar{\Gamma}_{i j} \triangleq\left[\begin{array}{cc}
\operatorname{sim}\left(-A_{i} M^{T}-B_{i} F_{j} M^{T}\right) & * \\
M-\mu A_{i} M^{T}-\mu B_{i} F_{j} M^{T} & \mu \operatorname{sim}(M)
\end{array}\right]
$$

Finalmente, as mudanças de variável a seguir são utilizadas:

$$
R \triangleq M^{T}, S_{j} \triangleq F_{j} M^{T}
$$

sendo possível escrever (31) da forma

$$
\begin{aligned}
\dot{V}(x) & =\sum_{i=1}^{r} \sum_{j=1}^{r} \beta_{i} \beta_{j} \xi^{T}\left(\Lambda_{i}+\bar{\Gamma}_{i j}\right) \xi \\
& =\sum_{i=1}^{r} \beta_{i}^{2} \xi^{T} \tilde{\Theta}_{i i} \xi+\sum_{i<j}^{r} \beta_{i} \beta_{j} \xi^{T} \bar{\Theta}_{i j} \xi
\end{aligned}
$$

sendo $\tilde{\Theta}_{i i}$ e $\bar{\Theta}_{i j}$ como em (30). Portanto, caso (27)(29) sejam satisfeitas garante-se que o sistema em malha fechada (26) é globalmente assintoticamente estável.

Uma vez que o multiplicador matricial $R$ foi introduzido nas LMIs, os ganhos podem ser obtidos de forma independente das matrizes de $P(x)$, i.e., eles são unicamente determinados fazendo-se $F_{i}=S_{i} R^{-1}$.

Ao contrário da condição LMI para síntese apresentada em (de Avellar et al., 2008), não se requer uma transformação de similaridade sobre as matrizes de $P(x)$. Isso pode tornar as condições propostas em (de Avellar et al., 2008) menos conservadoras. Por exemplo, considere matrizes $P_{1}$ e $P_{2}$ com elementos fora da diagonal iguais. No caso das condições apresentadas em (de Avellar et al., 2008), deve-se escolher adequadamente a matriz $R$ que aplicada nas transformações $T_{1}=R P_{1} R^{T}$ e $T_{2}=R P_{2} R^{T}$ ainda assegure que os termos fora da diagonal em $T_{1}$ e $T_{2}$ permaneçam iguais. Já no Teorema 4 , a matriz $R$ pode ser uma matriz não-singular qualquer.

Além dessa diferença, é possível aprimorar as condições propostas adicionando matrizes de folga, como foi realizado no Teorema 3.

Teorema 5 Considere um escalar $\mu>0$ dado. $O$ sistema (26) é estabilizável pelo controlador (25), com ganhos locais dados por $F_{i}=S_{i} R_{i}^{-1}$, se existirem matrizes
$P_{i}$, com a estrutura dada em (6), matrizes simétricas $Y_{i i}$ e matrizes $R_{i}, S_{i}$ e $Y_{j i}=Y_{i j}^{T}$ quaisquer tais que as LMIs abaixo sejam factíveis:

$$
\begin{aligned}
& P_{i} \succ 0, \\
& \tilde{\Theta}_{i i}, \prec Y_{i i} \\
& \bar{\Theta}_{i j}, \prec Y_{i j}+Y_{j i}, \quad i, j \in \mathcal{R}, i<j \\
& \bar{Y}=\left[\begin{array}{cccc}
Y_{11} & Y_{21}^{T} & \cdots & Y_{r 1}^{T} \\
Y_{21} & Y_{22} & \cdots & Y_{r 2}^{T} \\
\vdots & \vdots & \ddots & \vdots \\
Y_{r 1} & Y_{r 2} & \cdots & Y_{r r}
\end{array}\right] \prec 0
\end{aligned}
$$

sendo $\tilde{\Theta}_{i j}$ e $\bar{\Theta}_{i j}$ definidas em (30) e (31), respectivamente.

Prova: A demonstração segue passos similares aos da demonstração do Teorema 3, contudo tomando (35) como ponto de partida.

Nota 2 Nos Teoremas 4 e 5 o escalar $\mu$ deve ser obtido por meio de uma busca unidimensional.

\section{COMPARAÇÕES PARA ESTABILIZA- ÇÃO}

Nas seções seguintes, os métodos propostos para projeto de controlador PDC são aplicados em exemplos numéricos, no intuito de compará-los com outros métodos da Literatura.

Considere um sistema modelado pelas seguintes regras:

$$
\begin{array}{lll}
R_{1}: & \text { SE } & x_{2}(t) \text { é } \mathcal{M}_{2}^{1} \\
& \text { ENTÃo } & \dot{x}(t)=A_{1} x(t)+B_{1} u(t) \\
& & \\
R_{2}: & \text { SE } & x_{2}(t) \text { é } \mathcal{M}_{2}^{2} \\
& \text { ENTÃo } & \dot{x}(t)=A_{2} x(t)+B_{2} u(t)
\end{array}
$$

sendo que as matrizes locais são dadas por

$$
\begin{gathered}
A_{1}=\left[\begin{array}{ll}
3,6 & -1,6 \\
6,2 & -4,3
\end{array}\right], \quad A_{2}=\left[\begin{array}{ll}
-a & -1,6 \\
6,2 & -4,3
\end{array}\right] \\
B_{1}=\left[\begin{array}{c}
-0,45 \\
-3
\end{array}\right], \quad B_{2}=\left[\begin{array}{l}
-b \\
-3
\end{array}\right]
\end{gathered}
$$

De acordo com (6), as matrizes da função de Lyapunov devem ser :

$$
P_{1}=\left[\begin{array}{ll}
p_{11} & p_{21} \\
p_{21} & d_{22}^{1}
\end{array}\right], P_{2}=\left[\begin{array}{ll}
p_{11} & p_{21} \\
p_{21} & d_{22}^{2}
\end{array}\right]
$$


Os Teoremas 4 e 5 são aplicados para verificar a região estabilizável quando $a \in[0,25]$ e $b \in[0,2,5]$ considerando $\mu=0,025$. Os resultados são comparados com o Teorema 2 de (Montagner et al., 2009), usando $g=d=5$, resultando na Figura 2. Note que os resultados dos Teoremas 4 e 5 abrangem o resultado quadrático, sendo que o Teorema 5 é menos conservador que o Teorema 4 (para a malha de parâmetros considerada, 4 pontos a mais foram obtidos).

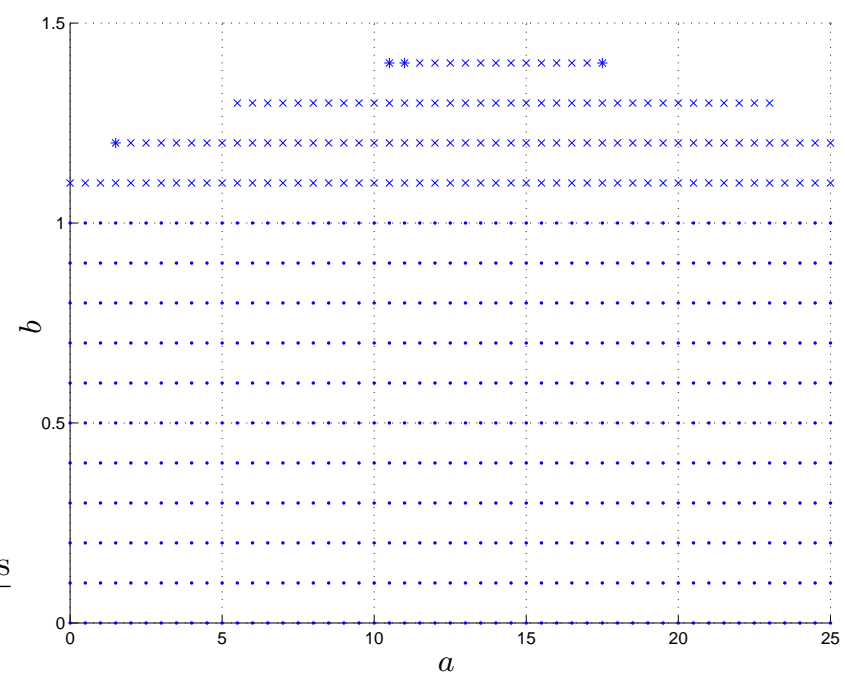

Figura 2: Regiões estabiliáveis geradas pelas abordagens baseadas em FLQ e FLF. (Montagner et al., 2009) $\rightarrow(\cdot)$; Teorema 4 $\rightarrow(\times)$ e $(\cdot)$; Teorema 5 inclui todos os pontos assinalados na Figura.

Para efeito de comparação considere os parâmetros $a=$ 5 e $b=1$, para os quais todas as abordagens foram factíveis. A abordagem em (Montagner et al., 2009) resulta em uma única matriz para a função quadrática $x^{T} P x$ :

$$
P=\left[\begin{array}{ll}
0,1406 & 0,4057 \\
0,4057 & 1,1969
\end{array}\right]
$$

e os ganhos do controlador PDC são

$$
F_{1}=\left[\begin{array}{ll}
-28,5890 & 9,2488
\end{array}\right], F_{2}=\left[\begin{array}{ll}
-353,343 & 119,323
\end{array}\right] \text {. }
$$

Para esse mesmos pârametros $a$ e $b$, o Teorema 4 gera as matrizes a seguir para a função de Lyapunov em (4):

$$
P_{1}=\left[\begin{array}{ll}
0,0421 & 0,1183 \\
0,1183 & 0,4156
\end{array}\right], P_{2}=\left[\begin{array}{ll}
0,0421 & 0,1183 \\
0,1183 & 0,3679
\end{array}\right]
$$

com os seguintes ganhos para (25):

$F_{1}=\left[\begin{array}{ll}-16,3195 & 4,7497\end{array}\right], F_{2}=\left[\begin{array}{ll}-53,9443 & 16,3159\end{array}\right]$.
Finalmente, para o Teorema 5 tem-se que

$$
P_{1}=\left[\begin{array}{ll}
0,0588 & 0,1627 \\
0,1627 & 0,5731
\end{array}\right], P_{2}=\left[\begin{array}{ll}
0,0588 & 0,1627 \\
0,1627 & 0,5053
\end{array}\right],
$$

com os ganhos

$F_{1}=\left[\begin{array}{ll}-18,9340 & 5,6639\end{array}\right], F_{2}=\left[\begin{array}{ll}-54,6945 & 16,7258\end{array}\right]$.

\section{EXEMPLO DE CONTROLE}

Considere um sistema fuzzy TS formado por duas regras:

$$
\begin{aligned}
& A_{1}=\left[\begin{array}{cc}
2 & -10 \\
2 & 0
\end{array}\right], B_{1}=\left[\begin{array}{l}
1 \\
1
\end{array}\right], \\
& A_{2}=\left[\begin{array}{cc}
2 & -5 \\
1 & 2
\end{array}\right], B_{2}=\left[\begin{array}{l}
1 \\
2
\end{array}\right]
\end{aligned}
$$

cujas funções de pertinência são:

$$
\begin{aligned}
& \beta_{1}\left(x_{1}(t)\right)=\left\{\begin{array}{cl}
{\left[1-\operatorname{sen}\left(x_{1}(t)\right)\right] / 2} & \text { se }\left|x_{1}(t)\right| \leq \pi / 2, \\
0 & \text { se } x_{1}(t)>\pi / 2, \\
1 & \text { se } x_{1}(t)<-\pi / 2
\end{array}\right. \\
& \beta_{2}\left(x_{1}(t)\right)=1-\beta_{1}\left(x_{1}(t)\right) .
\end{aligned}
$$

O vetor de estados é dado por $x(t)=\left[x_{1}(t) x_{2}(t)\right]^{T}$ com a variável premissa sendo apenas $x_{1}(t)$. O objetivo deste exemplo é obter controladores fuzzy PDC que estabilizem o sistema. Os ganhos obtidos através da aplicação do Teorema 4 usando $\mu=1$ são dados por:

$$
\begin{aligned}
& F_{1}=\left[\begin{array}{ll}
1,7371 & -10,4173
\end{array}\right], \\
& F_{2}=\left[\begin{array}{ll}
1,2429 & -6,3675
\end{array}\right] .
\end{aligned}
$$

As matrizes da FLF obtidas são dadas por:

$$
\begin{aligned}
& P_{1}=\left[\begin{array}{ll}
2,0388 & 0,4007 \\
0,4007 & 0,7993
\end{array}\right], \\
& P_{2}=\left[\begin{array}{ll}
0,7733 & 0,4007 \\
0,4007 & 0,7993
\end{array}\right] .
\end{aligned}
$$

Por outro lado, o Teorema 5 fornece os ganhos a seguir, quando $\mu=1$ :

$$
\begin{aligned}
& F_{1}=\left[\begin{array}{ll}
2,1676 & -11,9238
\end{array}\right], \\
& F_{2}=\left[\begin{array}{ll}
1,4037 & -7,0270
\end{array}\right] .
\end{aligned}
$$

A FLF obtida é dada segundo:

$$
\begin{aligned}
& P_{1}=\left[\begin{array}{ll}
1,2743 & 0,2339 \\
0,2339 & 0,4703
\end{array}\right], \\
& P_{2}=\left[\begin{array}{ll}
0,5324 & 0,2339 \\
0,2339 & 0,4703
\end{array}\right] .
\end{aligned}
$$


As Figuras 3 e 4 mostram o retrato de fases dos sistemas em malha aberta e em malha fechada com os ganhos obtidos via Teorema 4, respectivamente. Além disso mostram 4 trajetórias partindo de diferentes condições iniciais. Note que em malha fechada o campo vetorial converge para o ponto de equilíbrio, no caso a origem. Ambos controladores projetados são capazes de estabilizar o sistema TS, mas como o resultado obtido pelo Teorema 5 é muito similar ao obtido pelo Teorema 4, optou-se por omití-lo.

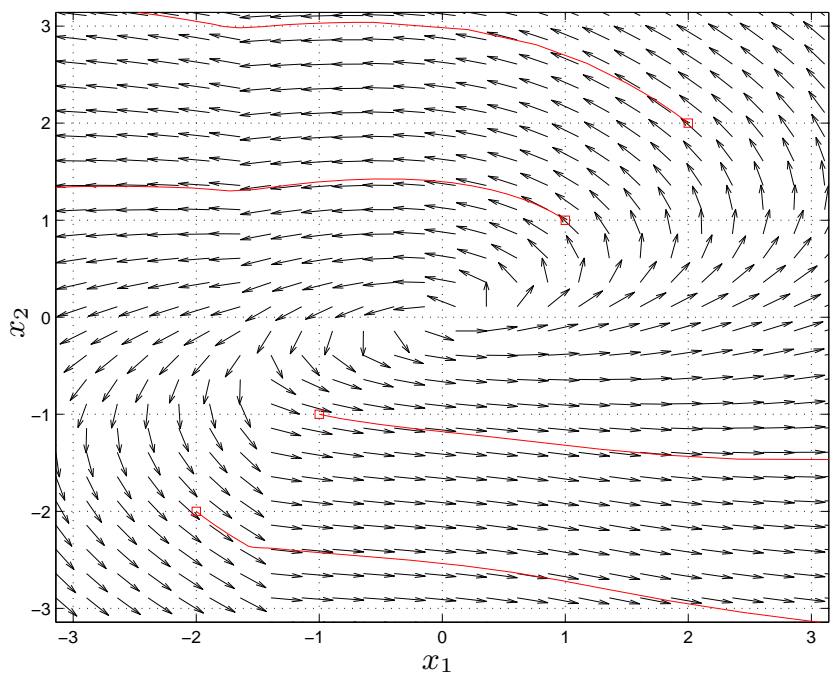

Figura 3: Diagrama de fase do sistema em malha aberta.

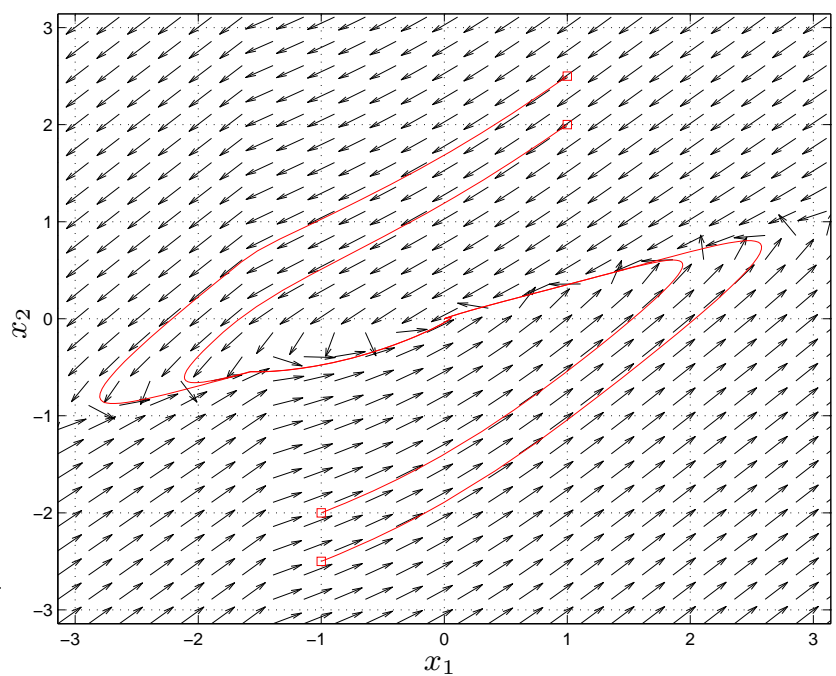

Figura 4: Diagrama de fase do sistema em malha fechada com o controlador PDC projetado segundo o Teorema 4.

\section{CONCLUSÕES}

Neste artigo foi apresentado um método sistemático para reduzir o conservadorismo presente na análise de estabilidade de sistemas TS contínuos. As novas condições LMIs são menos conservadoras que outros métodos da literatura baseados na FLQ e na FLF. Além disso, novas condições foram propostas para o projeto de controlador que são inteiramente baseadas em LMIs.

\section{AGRADECIMENTOS}

Os autores agradecem suporte financeiro do $\mathrm{CNPq}$ (Conselho Nacional de Desenvolvimento Científico e Tecnológico) e da FAPEMIG (Fundação de Amparo à Pesquisa do Estado de Minas Gerais).

\section{REFERÊNCIAS}

Andrea, C. Q., Pinto, J. O. P., Assunção, E., Teixeira, M. C. M. e Galotto Jr., L. (2008). Controle ótimo $\mathcal{H}_{\infty}$ de sistemas não-lineares com modelos fuzzy Takagi-Sugeno, Controle \& Automação 19(3): 256269.

Arrifano, N. S. D. e Oliveira, V. A. (2004). State feedback fuzzy-model-based control for Markovian jump nonlinear systems, Controle \& Automação 15(3): 279-290.

Arrifano, N. S. D., Oliveira, V. A. e Cossi, L. V. (2006). Synthesis of an LMI-based fuzzy control system with guaranteed cost performance: a piecewise Lyapunov approach, Controle $\&$ Automação 17(2): 213-225.

de Avellar, G. S. C., Mozelli, L. A. e Palhares, R. M. (2008). Condições LMIs alternativas para sistemas Takagi-Sugeno via função de Lyapunov fuzzy, Anais do CBA'2008, Juiz de Fora, MG, Brasil.

de Oliveira, M. (2004). Novos testes de estabilidade para sistemas lineares, Controle $\&$ Automação 15(1): $17-23$.

Fang, C.-H., Liu, Y.-S., Kau, S.-W., Hong, L. e Lee, C.-H. (2006). A new LMI-based approach to relaxed quadratic stabilization of T-S fuzzy control systems, IEEE Trans. Fuzzy Syst. 14(3): 386-397.

Gahinet, P., Nemirovski, A., Laub, A. J. e Chilali, M. (1995). LMI Control Toolbox User's Guide, 1 edn, The MathWorks Inc.

Haddad, W. M. e Chellaboina, V. (2008). Nonlinear dynamical systems and control: a Lyapunov-based approach, 1 edn, Princeton University Press. 
Johansson, M., Rantzer, A. e Årzén, K.-E. (1999). Piecewise quadratic stability of fuzzy systems, IEEE Trans. Fuzzy Syst. 7(8): 713-722.

Khiar, D., Lauber, J., Floquet, T., Colin, G., Guerra, T. e Chamaillard, Y. (2007). Robust Takagi-Sugeno fuzzy control of a spark ignition engine, Contr. Eng. Pract. 15(12): 1446 - 1456.

Kim, E. e Lee, H. (2000). New approaches to relaxed quadratic stability condition of fuzzy control systems, IEEE Trans. Fuzzy Syst. 8(5): 523-534.

Lian, K. Y., Wu, H. W. e Liou, J. J. (2006). Stability conditions for LMI-based fuzzy control from the viewpoint of membership functions, IEEE Trans. Fuzzy Syst. 14(6): 874-884.

Liu, X. e Zhang, Q. (2003). New approaches to $\mathcal{H}_{\infty}$ controller designs based on fuzzy observers for T-S fuzzy systems via LMI, Automatica 39(9): 15711582 .

Löfberg, J. (2004). Yalmip : A toolbox for modeling and optimization in MATLAB, Proc. of the CACSD, Taipei, Taiwan.

Montagner, V. F., Oliveira, R. C. L. F. e Peres, P. L. D. (2007). Necessary and sufficient LMI conditions to compute quadratically stabilizing state feedback controllers for Takagi-Sugeno systems, Proc. of $A C C$, New York, USA, pp. 4059-4064.

Montagner, V. F., Oliveira, R. C. L. F. e Peres, P. L. D. (2009). Convergent LMI relaxations for quadratic stabilizability and $\mathcal{H}_{\infty}$ control of Takagi-Sugeno fuzzy systems, IEEE Trans. Fuzzy Syst. 17(4): 863873.

Mozelli, L. A., Campos, C. D., Palhares, R. M., Tôrres, L. A. B. e Mendes, E. M. A. M. (2007). Chaotic synchronization and information transmission experiments: a fuzzy relaxed $\mathcal{H}_{\infty}$ control approach, Circ. Syst. Signal Process. 26(4): 427-449.

Mozelli, L. A., Palhares, R. M. e de Avellar, G. S. C. (2008). Novas condições de estabilidade e de estabilização para sistemas Takagi-Sugeno baseadas na função de Lyapunov fuzzy, Anais CBA'2008, Juiz de Fora, MG, Brasil.

Mozelli, L. A., Palhares, R. M., Souza, F. O. e Mendes, E. M. A. M. (2009). Reducing conservativeness in recent stability conditions of TS fuzzy systems, $A u$ tomatica 45(6): 1580 - 1583.

Rhee, B.-J. e Won, S. (2006). A new fuzzy Lyapunov function approach for a Takagi-Sugeno fuzzy control system design, Fuzzy Set. Syst. 157(9): 12111228 .
Sala, A. e Ariño, C. (2007). Asymptotically necessary and sufficient conditions for stability and performance in fuzzy control: applications of Polya's theorem, Fuzzy Set. Syst. 158(24): 2671-2686.

Souza, F. O., Palhares, R. M. e Ekel, P. Y. (2008). Análise de estabilidade assintótica e exponencial em redes neurais artificiais sujeitas a retardo no tempo e a incertezas do tipo politópicas, Controle 86 Automação 19(2): 116-127.

Souza, F. O., Palhares, R. M. e Ekel, P. Y. (2009). Improved asymptotic stability analysis for uncertain delayed state neural networks, Chaos, Solitons and Fractals 39(1): 240-247.

Souza, F. O., Palhares, R. M., Mendes, E. M. A. M. e Tôrres, L. A. B. (2008a). Further results on masterslave synchronization of general Lur'e systems with time-varying delay, Int. J. Bifurcat. Chaos Appl. Sci. Eng. 18(1): 187-202.

Souza, F. O., Palhares, R. M., Mendes, E. M. A. M. e Tôrres, L. A. B. (2008b). Robust $H_{\infty}$ control for master-slave synchronization of Lur'e systems with time-delay feedback control, Int. J. Bifurcat. Chaos Appl. Sci. Eng. 18(4): 1161-1173.

Takagi, T. e Sugeno, M. (1985). Fuzzy identification of systems and its applications to modeling and control, IEEE Trans. Syst., Man, Cybern. 15(1): 116132 .

Tanaka, K., Hori, T. e Wang, H. O. (2003). A multiple Lyapunov function approach to stabilization of fuzzy control systems, IEEE Trans. Fuzzy Syst. 11(4): 528-529.

Tanaka, K., Ikeda, T. e Wang, H. O. (1998). Fuzzy regulators and fuzzy observers: relaxed stability conditions and LMI-based designs, IEEE Trans. Fuzzy Syst. 6(2): 250-264.

Tanaka, K., Ohtake, H. e Wang, H. O. (2007). A descriptor system approach to fuzzy control system design via fuzzy Lyapunov functions, IEEE Trans. Fuzzy Syst. 15(3): 333-341.

Tanaka, K. e Sugeno, M. (1992). Stability analysis and design of fuzzy control systems, Fuzzy Set. Syst. 45(2): 135-156.

Tanaka, K. e Wang, H. O. (2001). Fuzzy Control Systems Design and Analysis: A Linear Matrix Inequality Approach, John Wiley \& Sons. 
Tanscheit, R., Gomide, F. e Teixeira, M. C. M. (2007). Modelagem e controle nebuloso, in L. A. Aguirre (ed.), Enciclopédia de Automática: Controle $\&$ Automação, Vol. 3, Blucher, pp. 283-324.

Teixeira, M. C. M. e Assunção, E. (2007). Extensões para sistemas não-lineares, in L. A. Aguirre (ed.), Enciclopédia de Automática: Controle $\&$ Automação, Vol. 1, Blucher, pp. 218-246.
Teixeira, M. C. M., Assunção, E. e Avellar, R. G. (2003). On relaxed LMI-based designs for fuzzy regulators and fuzzy observers, IEEE Trans. Fuzzy Syst. 11(5): 613-623.

Teixeira, M. C. M., Pietrobom, H. C. e Assunção, E. (2000). Novos resultados sobre a estabilidade e controle de sistemas não-lineares utilizando modelos fuzzy e LMI, Controle \& Automação 11(1): 37-48. 